\title{
Long noncoding RNAs involvement in Epstein-Barr virus infection and tumorigenesis
}

\author{
Jing Zhang ${ }^{1,2,3}$, Xiaohan $\mathrm{Li}^{1,2,3}$, Jingjin $\mathrm{Hu}^{1,2,3}$, Pengfei $\mathrm{CaO}^{2}$, Qijia Yan ${ }^{1,2}$, Siwei Zhang ${ }^{1,2,3}$, Wei Dang ${ }^{1,2,3}$ and \\ Jianhong $L u^{1,2^{*}}$
}

\begin{abstract}
The Epstein-Barr virus (EBV) is a ubiquitous $\gamma$-herpesvirus related to various types of cancers, including epithelial nasopharyngeal carcinoma, gastric carcinoma, and lymphoma. Long noncoding RNAs (IncRNAs) are expressed extensively in mammalian cells and play crucial roles in regulating various cellular processes and multiple cancers. Cellular IncRNAs can be differentially expressed induced by EBV infection. The dysregulated IncRNAs probably modulate the host immune response and other biological functions. At present, IncRNAs have been found to be significantly increased or decreased in EBV-infected cells, exosomes and EBV-associated cancers, suggesting their potential function and clinical application as biomarkers. In addition, EBV-encoded IncRNAs, BART and BHLF1 IncRNAs, may play roles in the viral oncogenesis. Analysis of the specific IncRNAs involved in interactions with the EBV machinery will provide information on their potential mechanism of action during multiple steps of EBV tumorigenesis. Here, we review the current knowledge regarding EBVrelated IncRNAs and their possible roles in the pathogenesis of EBV-associated cancers.
\end{abstract}

Keywords: Long noncoding RNA, Epstein-Barr virus, Tumorigenesis, Epithelial cancer, Lymphoma

\section{Background}

Epstein-Barr virus (EBV) is a ubiquitous $\gamma$-herpesvirus, which is the first confirmed human tumor virus [1]. EBV is able to establish latent infection in B lymphocytes and other types of cells such as T, NK, and some epithelial cells. Primary EBV infection typically appears in childhood as a symptomless or mild infection. The establishment of viral latency is vital to maintain lifelong persistence of the virus inside the infected cells. In the latent status, EBV exhibits a severely restricted gene expression profile for the purpose of avoiding host immune surveillance [1, 2]. EBV infection is associated with multiple diseases such as Burkett's

\footnotetext{
* Correspondence: jianhlu@csu.edu.cn

${ }^{1} \mathrm{NHC}$ Key Laboratory of Carcinogenesis, Department of Pathology, Xiangya Hospital, Central South University, Changsha 410080, China

${ }^{2}$ The Key Laboratory of Carcinogenesis and Cancer Invasion of the Chinese Ministry of Education, Department of Hematology, Xiangya Hospital, Central South University, Changsha 410080, China

Full list of author information is available at the end of the article
}

lymphoma, nasopharyngeal carcinoma (NPC), gastric cancer (GC), Hodgkin's disease and breast cancer [1, 2].

In human genomes, fewer than $2 \%$ of genes are transcribed into mRNAs [3]. Emerging evidence has shown that many noncoding RNAs (ncRNAs) also play important cellular functions. Long noncoding RNAs (lncRNAs) are defined as RNAs over 200 nucleotides in length without protein-coding ability and have essential functions for a range of biological processes [4]. LncRNAs are mainly the transcribed and spliced products of RNA polymerase II transcription. They are usually $5^{\prime}$ capped and may contain a polyadenylated tail at the $3^{\prime}$ end. LncRNAs have been confirmed to impact cancer initiation, development and progression and utilize a multitude of mechanisms mediated by specific sequences or structural motifs that bind DNA, RNA, or proteins [4, 5]. According to their position relative to neighboring protein-coding genes, lncRNAs are classified as sense, 
antisense, bidirectional, intronic, or intergenic. The human genome encodes thousands of IncRNAs that were previously considered 'dark matter' or 'junk' in the genome. However, recent studies have illuminated the roles of lncRNAs, and they are now considered important physiological regulators of cell homeostasis, growth, and differentiation $[4,5]$. Now lncRNAs have been shown to play an elegant role in regulating gene expression at various levels, and thus in affecting aspects of cellular homeostasis including proliferation, survival, migration and genomic stability [5-7]. Collective evidence has also identified the important role of lncRNAs in regulating antiviral responses $[8,9]$.

In structure, lncRNAs are similar to mRNAs. While, unlike mRNAs which are normally cytoplasmic, lncRNAs show various subcellular distributions that can be detected in the nucleus, cytoplasm, or both. Moreover, lncRNAs are usually tissue specific and less conserved than mRNAs $[6,10]$. Some lncRNAs interact with the target genes and regulating their expression, thus mediating cell signaling pathways $[5,6,11]$. Current researches indicate that lncRNAs are dysregulated in the infection of viruses. Howerver, the studies of lncRNAs in this field are still in infancy with regard to their functions. Some lncRNAs can serve as scaffolds to regulate the transport or posttranslational modification of proteins, as well as play an important regulatory role during viral infection such as triggering the antiviral reaction $[8,12]$.

This review highlights IncRNAs associated with EBV infection including EBV-related host lncRNAs and EBVencoded lncRNAs, focusing especially on their expression patterns and functions.

\section{Main text}

\section{EBV-regulated host IncRNAs}

In recent years, lncRNAs have been shown to functionally exert both positive and negative effects on innate immunity and antiviral regulators. Some host lncRNAs show differential expression during EBV infection (Table 1), indicating their importance in the host response to EBV infection. The aberrantly expressed host lncRNAs, designated as EBV-regulated lncRNAs, can be regulated by or interacted with EBV-encoded proteins and microRNAs [22].

\section{EBV-regulated host IncRNAs may play roles in epithelial carcinomas}

EBV infection contributes to a variety of human tumors, including epithelial carcinomas. NPC is a kind of cancer derived from epithelial cells. It is the most common type of head and neck tumor in Asia, especially southern China, and parts of Africa. Because the symptoms are insidious at the onset of NPC, $75-90 \%$ of NPC cases are not diagnosed until an advanced stage, which has a high rate of relapse [11, 23, 24]. Moreover, treatment for patients with NPC is not satisfactory, resulting in a low five-year survival rate and a high recurrence rate [24-26]. Considering the current situation, it is important to characterize sensitive and specific biomarkers for the early diagnosis of high-risk individuals as well as the evaluation of NPC therapeutic effects. EBV infection is closely associated with NPC and is present in almost $100 \%$ of low or non-differentiated NPC cases [1, 2]. EBV can establish latent infection in NPC that leads to changes in both intracellular metabolism and molecular expression $[1,2,27]$. Therefore, EBV-related pathogenesis, biomarkers and therapeutics for NPC are worth exploring.

Persistent latent EBV infection has been found among almost all NPC patients, indicating its etiological role in the initiation and progression of NPC. It is necessary to identify potential biomarkers for the early-stage diagnosis, progression and prognosis of NPC. According to a previous study by Xiao-xiao Li et al., the application of nextgeneration sequencing technology to 7 NPC tissue samples and 7 normal nasopharyngeal tissue samples revealed that 2192 lncRNAs were abnormally expressed in NPC and that 62 lncRNAs trans-regulated genes were involved in EBV infection [13]. In particular, several lncRNAs were downregulated, including lncRNA-NR2F2 antisense RNA1 (NR2F21), lncRNA-family with sequence similarity 95 member C (FAM95C), and long intergenic nonprotein coding RNA 1106 (LINC01106), and some were upregulated, including lncRNA-CH507-513H4.6, IncRNA-TH AP9 antisense RNA 1 (THAP9-AS1), IncRNACH507-513 H4.3 and IncRNA-RP4-794H19.1 [13]. Sixty two genes potentially trans-regulated by the IncRNAs such as CD44 (Hyaluronan/CD44 signaling plays an important role in head and neck squamous cell carcinoma progression) and interleukin 1 receptor associated kinase 1 (IRAK1) are involved in the EBV infection pathway [13]. However, only a few known lncRNAs have been functionally annotated. Therefore, lncRNA-related pathogenesis, biomarkers and therapeutics for NPC caused by EBV are worth further exploring.

Moreover, LINC00312, also known as NPC associated gene 7 (NAG7), is one of the earliest studied lncRNAs and acts as a tumor suppressor gene in NPC [28]. LINC00312 expression was significantly different between NPC epithelium and various noncancerous nasopharyngeal epithelia. LINC00312 can restrict the proliferation of NPC cells and prevent the progression from $\mathrm{G} 1$ to $\mathrm{S}$ phase in the cell cycle, thus aggravating cell apoptosis. The expression of LINC00312 is negatively related to EBV-encoded noncoding RNA, EBER1 positively correlated with lymph node metastasis of NPC. At the same time, relatively low or high expression of LINC00312 can be used for the distinction of NPC prognosis prediction with lymph node or non-lymphatic metastasis [28]. Therefore, LINC00312, may serve as a potential target in the diagnosis and therapy for EBV associated NPC. 
Table 1 Cellular IncRNAs regulated by EBV in the infection and associated cancers

\begin{tabular}{|c|c|c|c|}
\hline IncRNAs & cancer/cell types & regulation status & references \\
\hline NR2F21 & NPC & down & [13] \\
\hline FAM95C & NPC & down & [13] \\
\hline LINC01106 & NPC & down & [13] \\
\hline CH507-513H4.6 & NPC & up & [13] \\
\hline HAP9-AS1 & NPC & up & [13] \\
\hline $\mathrm{CH} 507-513 \mathrm{H} 4.3$ & NPC & up & [13] \\
\hline RP4-794H19.1 & NPC & up & [13] \\
\hline LINC00312(NAG7) & NPC & down & [13] \\
\hline LOC553103 & NPC & down & {$[14]$} \\
\hline APAF1-AS1 & NPC & up & {$[15]$} \\
\hline AL359062 & NPC & up & {$[15]$} \\
\hline SNHG8 & EBVaGC & up & {$[16]$} \\
\hline RP5-1039 K5.19 & EBVaGC & up & {$[17]$} \\
\hline TP73-AS1 & EBVaGC & up & {$[17]$} \\
\hline \multirow[t]{4}{*}{ MALAT1 } & NPC & up & {$[15]$} \\
\hline & 293-EBV, AGS-EBV & up & [18] \\
\hline & NPC C666-1 & down & {$[19,20]$} \\
\hline & diffuse large B cell lymphoma & up & \\
\hline BC200 & 293-EBV, AGS-EBV, C666-1 & up & [18] \\
\hline LINC00672 & 293-EBV, AGS-EBV, C666-1 & up & [18] \\
\hline \multirow[t]{2}{*}{ LINC00982 } & 293-EBV, C666-1 & up & {$[18]$} \\
\hline & AGS-EBV & down & [18] \\
\hline LINC02067 & $\begin{array}{l}\text { 293-EBV, AGS-EBV } \\
\text { C666-1 }\end{array}$ & $\begin{array}{l}\text { down } \\
\text { no change }\end{array}$ & {$[18]$} \\
\hline \multirow[t]{2}{*}{ LOC100128494 } & 293-EBV & down & {$[18]$} \\
\hline & AGS-EBV, C666-1 & up & {$[18]$} \\
\hline LOC100505716 & 293-EBV, AGS-EBV, C666-1 & down & {$[18]$} \\
\hline IGFBP7-AS1 & 293-EBV & down & [18] \\
\hline CYTOR & $\mathrm{LCL}$ & up & {$[20]$} \\
\hline NORAD & $\mathrm{LCL}$ & down & {$[20]$} \\
\hline $7 S L$ & $\mathrm{LCL}$ and exosomes & up & {$[21]$} \\
\hline $\mathrm{H} 19$ & LCL and exosomes & up & {$[21]$} \\
\hline H19-AS & LCL and exosomes & up & {$[21]$} \\
\hline
\end{tabular}

NPC Nasopharyngeal carcinoma, EBVaGC EBV associated gastric carcinoma, LCL Lymphoblastoid cell line

Interestingly, EBV encodes at least 44 mature microRNAs (miRNAs), and these miRNAs can promote the occurrence and progression of many tumors by directly targeting both host genes and viral genes including some lncRNAs which contain miRNA-binding sites $[2,29]$. According to a tissue microarray analysis, EBV-miRBART6-3p can directly target and downregulate lncRNA LOC553103. The overexpression of LOC553103 can promote tumor proliferation, invasion, mesenchymal transition (EMT), and metastasis in vitro and in vivo [14]. The results undoubtedly illustrate the potential application of LOC553103 in the treatment of NPC. The recognition of cross-talk between viral miRNAs and lncRNAs provides novel insight into the influence of EBV infection on tumorigenesis.

Emerging studies show that lncRNAs may be unloaded into the blood by serum extracellular vesicles such as microvesicles and exosomes that are regarded as vehicles of cell-cell communication [5, 30]. The microvesicles transfer viral and cellular factors, particularly in the case of persistent infections such as those of the herpesviruses, and allow the virus to respond to or control the cellular microenvironment, which could be beneficial to both the virus and host. The lncRNAs released into the 
blood can be quantitatively analyzed [5, 31]. Therefore, lncRNAs released from tumor cells can serve as potential liquid biopsy biomarkers by virtue of their distinct characteristics [5, 30]. For example, the lncRNAs MALAT1, APAF1-AS1, and AL359062 are significantly upregulated in the serum circulation of patients with NPC [15]. These lncRNAs increase in number after NP69 cells (EBV-negative normal nasopharyngeal epithelial cells) are infected with EBV. In addition, the circulating lncRNAs MALAT1, APAF1-AS1, and AL359062 are also associated with poor prognosis in patients with EBV-associated NPC, suggesting their application as biomarkers for the prognasis of NPC [15]. The findings undoubtedly broaden the lncRNAs landscape in NPC and shed light on the roles of lncRNAs, which would be conductive to the comprehensive management of NPC.

Gastric Cancer (GC) is another type of epithelial carcinoma and is the fourth most common cancer worldwide, and the second leading cause of cancer death. GC is a complicated disease with high heterogeneity. EBV is considered to have an important causal role in GC $[1,2,32]$; thus, one kind of $\mathrm{GC}$ is $\mathrm{EBV}$-associated gastric carcinoma (EBVaGC), which is ulcerative and mainly occurs in the cardia and gastric body. EBVaGC is a unique subtype of GC, accounting for approximately $10 \%$ of all cases of GC. EBV contributes to the deregulation of host lncRNAs that may play a pivotal regulatory role in human GC. The expression of small nucleolar RNA host gene 8 (SNHG8) in cultured EBV positive GC cells is significantly higher than that in noncancerous gastric mucosa cells or EBV-negative gastric cancer tissues [33, 34]. The expression of SNHG8 in EBVaGC is significantly correlated with tumor-nodemetastasis (TNM) stage [34]. Further study shows that SNHG8 interacts with EBV genes, including BHLF1, LF3, BHRF1 and BNLF2a. It is predicted that SNHG8 can modulate the expression of TRIM28, EIF4A2, NAP1L1, PLD3, RPL18A, and TRPM7, which may have direct effects on the stomach. The study also demonstrates that the downregulation of SNHG8 inhibits cell growth, arrest the cell cycle and facilitate apoptosis. A recent study by Jing Liu et al. also revealed that the knockdown of SNHG8 with specific shRNAs inhibited cell proliferation and colony formation, arrested the cell cycle in the G0/G1 phase in vitro and suppressed tumor growth in vivo [16]. Thus, SNHG8 may function as an oncogene and participate in the development of EBVaGC, and this knowledge provides information for the treatment and prognosis of EBVaGC.

LncRNAs can play roles through regulating their target genes. It has been previously validated that lncRNAs and microRNAs function as competing endogenous RNAs (ceRNAs) and suppress each other, thus forming a regulatory ceRNA network (lncRNAs-miRNAs-mRNAs) that regulates target mRNAs [5-7]. In a study by Jing-jing Jing et al. [17], the authors used the integration of multilevel expression data and a bioinformatics approach to identify key elements and interactions involved in EBVaGC and to establish a regulatory ceRNA network involving these key elements of EBVaGC. Overlapping genes and regulators such as CXCL10, GDF5, PTGER3, SMAD5, miR-68773p, RP5-1039 K5.19, TP73-AS1, EBV-miR-BART1-3p and EBV-miR-BART22 were observed in diverse regulation networks. EBV-related miRNAs EBV-miR-BART1-3p and EBV-mir-BART22 were shown to regulate CXCL10 and SMAD5. In particular, two unreported lncRNAs, RP51039 K5.19 and TP73-AS1, were identified in the ceRNA regulation network, which possibly provides a new avenue for the in-depth study of EBVaGC [17].

In addition, our recent research based on genomewide RNA sequencing revealed differential IncRNA expression profiles in EBV genome-infected human embryonated kidney cells (293-EBV), and identified the expression patterns of eight lncRNAs (LncRNA-BC200, MALAT1, LINC00672, LINC00982, IGFBP7-AS1, LOC100128494, LINC02067, LOC100505716) in EBVpositive NPC (C666-1) and GC cell lines (AGS-EBV) (Table 1) [18]. This study emphasized lncRNAs-specific functional alterations possibly influenced by EBV infection. We also explored the potential target genes of the lncRNAs. Interesting, the lncRNAs have some potentially common target genes such as eIF4A3, FUS, and UPF1. The exact function and mechanism of lncRNAs involved in EBV tumorigenesis are underway of investigation by us. The databases of lncRNAs in cancers might be connected with tumorigenic phenotypes in the further investigation. However, we should aware the fact that the expression patterns of one lncRNA may be inconsistent in different cancers and cell types (Table 1), implying their different roles in different cancers. The differentially expressed lncRNAs could be also developed as potential biomarkers in the diagnosis and therapy of EBV-associated NPC or GC.

\section{EBV-regulated host IncRNAs are involved in lymphomagenesis}

EBV is latent in B lymphocytes throughout the host lifetime once it invades and cannot be cleared by the host. Latent EBV infection is related to human lymphoid diseases including Burkitt's lymphoma, Hodgkin's lymphoma, and posttransplant lymphoproliferative diseases [1]. EBV infection can induce B lymphocyte proliferation, immortalization, or immune escape in vitro and in vivo $[1,2]$. The human EBV-transformed lymphoblastoid cell line (LCL), which is obtained by infecting peripheral blood monocular cells (PBMCs) or primary human resting B lymphocytes (RBLs) with EBV derived from the B95-8 cell lines, has been used extensively for human genetic, pharmacogenomic, and immunologic studies. 
In a recent study, the lncRNAs and anti-sense RNAs expressed differentially in RBLs infected with B95.8 strain derived EBV were analyzed by RNA-seq [20]. This study provided the dynamic expression patterns of a series of lncRNAs during EBV infection. There are 26 lncRNAs immediately up-regulated and 33 lncRNAs going down immediately upon EBV infection. Some of them may change the expression status with infection time. In the present RNA-seq investigation, CYTOR and NORAD lncRNAs were found to be important for LCL growth and survival [20]. NORAD can bind to proteins involved in DNA replication and repair [35].

According to gene expression analysis of the LCLs, the lncRNAs 7SL, H19, and H19-AS as well as p53 mRNA showed higher expression in the autologous LCL than in the PBMCs [21]. These lncRNAs are implicated in the development of many other tumors, suggesting that they can promote the progression of EBV-induced tumorigenesis. Nine lncRNAs were found in the LCL exosome cargo: 7SL, H19, H19 upstream conserved 1\&2, H19 antisense, HAR1B, HOXA6as, NDM29, SNHG5, and Tsix. LncRNAs H19 and H19-AS were enriched in exosomes and their expression was upregulated, while the rest of the lncRNAs were downregulated and present at lower levels than H19 and H19-AS in exosomes. Some studies also suggest that exosomes released by EBVinfected cells may affect tumorigenesis, metastasis, the tumor microenvironment, immune escape and maintenance of viral physiology, in addition to affecting neighboring cells and playing a long-lasting role in infecting cells $[10,30]$. Due to the role that LCL exosomal cargo transfer might play in maintaining persistent infection and tumorigenesis, it is important to characterize the exosomal lncRNAs released from LCLs. EBV-regulated lncRNAs may frequently affect the expression of their neighboring genes through looping. It would be interesting to further characterize their roles in LCLs.

It is noticeable that LCLs are not stable cancer cells and the related study may not well reflect lymphomagenesis. Some lncRNAs such as MALAT1 may be expressed unstably with the time point of EBV infection in LCLs [20]. Whereas, MALAT1 upregulation can promote tumorigenesis and immune escape in diffuse large $\mathrm{B}$ cell lymphoma, which may be related to EBV infection [19]. MALAT1 is also a tolerance regulator in immunity that has important implications in the induction of tolerogenic dendritic cells and regulatory $\mathrm{T}$ cells [36].

\section{EBV-encoded IncRNAs}

The viral infection generally changes the expression of the host lncRNAs, thus playing important regulatory roles on host genes. On the other hand, the IncRNA encoded by a virus can also modulate the expression of host genes which are critical for the infection and pathogenesis of the virus. EBV is recently found to encode its own lncRNAs from the viral genome.

\section{EBV-encoded BART IncRNAs}

In EBV-infected epithelial malignancies, including NPC and $\mathrm{GC}$, the transcription from the BamHI A region of the viral genome is extensive. The alternatively spliced transcripts are called the BamHI A rightward transcripts (BARTs). BARTs are expressed at high levels endogenously. In EBV-infected gastric tumors, 99\% of all virally derived polyadenylated transcripts are from BARTs [37]. These transcripts may result in the production of miRNAs or lncRNAs [2, 37, 38]. EBV was the first human virus identified to encode miRNAs that mapped to the BART regions of the genome $[2,39]$. Study has revealed that the BART miRNAs were differentially expressed, abundant among epithelial tissue while barely detectable in lymphoid cell lines [39]. The EBV encoded BART miRNAs have been validated to confer a significant and selective growth advantage to EBV positive tumor cells in vivo, including more efficient tumor seeding, larger tumors and higher and rapider mortality [38]. The function of spliced BART lncRNAs are under exploration by researchers. BART lncRNAs are located in the nucleus of EBV-infected cells. The BART lncRNAs knockdown significantly affects the expression of genes associated with cell adhesion, oxidoreductase activity, metastasis of NPC, inflammation, and immunity [40]. BART lncRNA appears to control Pol II at the promoter region and may thus regulate IFN-beta1 and CXCL8 expression in NPC [40].

The BART IncRNAs are also recently found to be regulated by NF- $\mathrm{B}$ signaling in the induction of EBV lytic replication, which is potentially an option for the treatment of EBV-associated carcinomas [11].

Both the BART miRNAs and the spliced or polyadenylated BART nuclear RNAs are derived from the same primary transcripts from the BamHI A region of the viral genome [39, 40]. These BART-derived transcripts are capable of regulating the expression of host cellular genes. The abundance of the BART RNAs and specific splicing patterns contribute to expression of the BART miRNAs. Impressively, Aron R. Marquitz's team presented the first evidence that the spliced BART transcripts functioned as lncRNAs independently of miRNA formation. They also confirmed that BART lncRNA could function in downregulating specific cellular genes, including tumor suppressors [40].

During latent EBV infection, BART miRNAs downregulate the some important tumor suppressors by targeting the 3' UTR of the genes, and thus altering the growth of tumor cells [2, 40]. Aron R. Marquitz's team revealed that the BART lncRNAs also play a significant role in the transcriptional reprogramming in latent EBV infection. RASA1 is a negative regulator of the RAS 


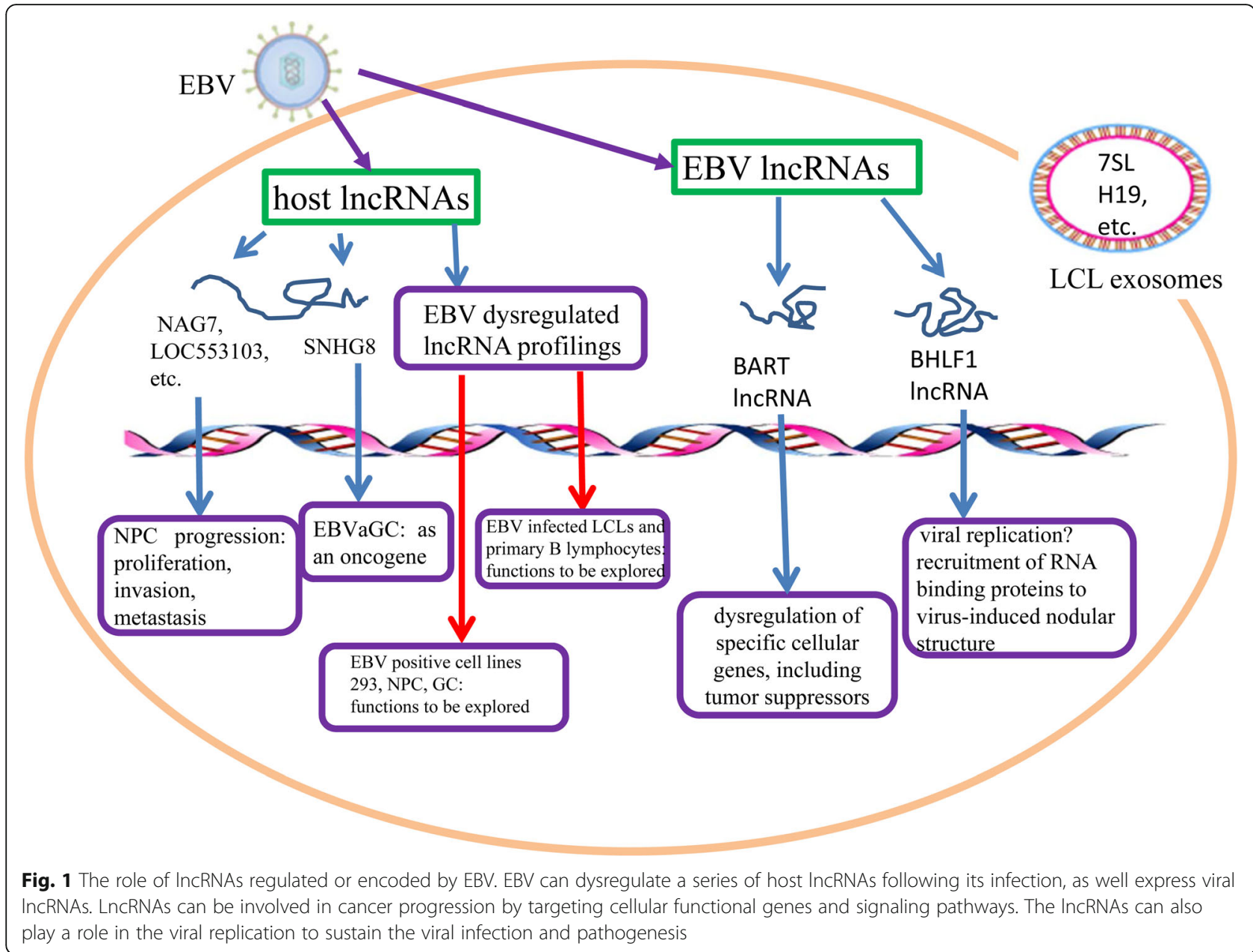

oncogene. The tumor suppressor RASA1 was downregulated in response to BART lncRNA expression without any response to the miRNA target gene assay [40]. This implies that it is the IncRNA rather than the miRNAs that is responsible for RASA1 regulation. Distinct function for the different spliced isoforms of the BART lncRNAs remains to be studied.

The RNA-seq analysis suggests BART lncRNAs regulate genes involved in the unfolded protein response (UPR) [41]. BART lncRNAs may have direct or indirect effects on the master regulatory transcription factors that make up the UPR, including XBP1, ATF4 and ATF6, which are likely responsible for the subsequent decrease in stress responsive genes in EBV tumorigenesis. It is possible that the extremely high level of $\mathrm{CpG}$ island promoter methylation in EBV-positive gastric carcinomas promotes the silencing of genes. Hence, it is speculated that the BART IncRNAs may have impact on histone regulation or potentially contribute to directing the DNA methylation phenotype. It is clear that several mechanisms of transcriptional regulation have been ascribed to lncRNAs. Taken together, BART IncRNAs can be important for viral oncogenesis. However, continued explorations are still required on how the BART lncRNAs downregulate genes during EBV latency.

\section{EBV-encoded BHLF1 IncRNAs}

BHLF1, a lytic-cycle gene expressed upon the initiation of latency, 1980 nucleotides in length and G-rich, also encodes a long non-coding RNA (lncRNA). BHLF1 has been shown to function as a lncRNA during the viral replicative cycle, where it contributes to the function of the origin of lytic replication (OriLyt) in cis by forming an RNA-DNA hybrid at the site of transcription [42]. The expression of BHLF1 protein depends on the presence of SM, another lytic-cycle protein [37]. BHLF1 lncRNA was localized to nodules only at the surface of viral replication compartments and was not found within the inner replication compartments [37]. According to Richard Park's report, during late lytic infection, BHLF1 lncRNA contains a series of tandem repeat sequences, which may play structural and scaffolding roles in recruitment of RNA-binding proteins to VINORCs (virus-induced nodular structures). Therefore, BHLF1 lncRNA may help the viral replication. 


\section{Conclusions}

In summary, cellular lncRNAs are expressed at different levels following EBV infection (Table 1). This is a new insight to help understand the role of viruses in modulating tumor progression (Fig. 1). EBV also encodes its own viral lncRNAs (BART lncRNAs and BHLF1 lncRNA) which may play important roles in oncogenesis and the regulation of some host functions. All the lncRNAs interacting with the host environment during infection potentially improve clinical treatment for EBV infection. In addition, the correlation between lncRNAs and EBVassociated cancers can be detected and analyzed in clinical patient samples. Emerging evidences have revealed the strong regulatory functions of lncRNAs in cancers. LncRNAs are also increasingly regarded as potential biomarkers in the diagnosis, prognosis, and treatment of neoplastic diseases, providing important basic support for medical solutions. However, lncRNAs related to EBV infection and tumorigenesis remain to be further investigated in their functional annotation and our better understanding.

\begin{abstract}
Abbreviations
BHLF1: BamHI H leftward reading frame 1; ceRNAs: competing endogenous RNAs; CYTOR: Cytoskeleton regulator RNA; EBV: Epstein-Barr virus; EBVaGC: Epstein-Barr virus-associated gastric carcinoma; elF4AIll: eukaryotic translation initiation factor 4A; FAM95C: IncRNA-family with sequence similarity 95 member C; GC: Gastric cancer; GO: Gene Ontology; IRAK1: Interleukin 1 receptor associated kinase 1; KEGG: Kyoto Encyclopedia of Genes and Genomes; LCL: Lymphoblastoid cell line; LF3: Leftward reading frame 3; LINC00672: Long intergenic non-protein coding RNA 672; LINC00982: Long intergenic non-protein coding RNA 982; LINC01106: Long intergenic nonprotein coding RNA 1106; LncRNA: Long-noncoding RNA; LncRNA-BC200: BCYRN1, brain cytoplasmic RNA 1; MALAT1: Metastasis associated lung adenocarcinoma transcript 1; MIR17HG: mir-17-92a-1 cluster host gene; mRNA: messenger RNA; NAG7: NPC associated gene 7; NPC: Nasopharyngeal carcinoma; NR2F21: IncRNA-NR2F2 antisense RNA1; PBMCs: Peripheral blood monocular cells; qRT-PCR: the quantitative real time RT-PCR; THAP9-AS1: InCRNA-THAP9 antisense RNA 1; TNM: Tumor-nodemetastasis; UPR: Unfolded protein response; VINORCs: Virus-induced nodular structures
\end{abstract}

\section{Acknowledgments}

All authors contributed to this manuscript and approved the final manuscript. This work was supported by the instruction of J.L and The Key Laboratory of Carcinogenesis and Cancer Invasion of the Chinese Ministry of Education, Xiangya Hospital, School of Basic Medical Science, Central South University.

\section{Authors' contributions}

$J Z$ and $X L$ performed the selection of literature, drafted the manuscript, and prepared the figure and table. JH, PC, QY, SWZ, and WD collected the related references and participation in discussion. QY and $J$ designed this review and revised the manuscript. All authors contributed to this manuscript. All authors read and approved the final manuscript.

\section{Funding}

This work was supported by the National Natural Science Foundations of China (31670171, 81974427).

\section{Availability of data and materials}

Not applicable.

Ethics approval and consent to participate Not applicable.

\section{Consent for publication}

All authors have agreed on the consent of the manuscript.

\section{Competing interests}

The authors declare that they have no competing interests.

\section{Author details}

${ }^{1} \mathrm{NHC}$ Key Laboratory of Carcinogenesis, Department of Pathology, Xiangya Hospital, Central South University, Changsha 410080, China. ${ }^{2}$ The Key Laboratory of Carcinogenesis and Cancer Invasion of the Chinese Ministry of Education, Department of Hematology, Xiangya Hospital, Central South University, Changsha 410080, China. ${ }^{3}$ Department of Microbiology, School of Basic Medical Science, Central South University, Changsha 410078, China.

Received: 22 October 2019 Accepted: 27 February 2020

Published online: 09 April 2020

\section{References}

1. Young LS, Rickinson AB. Epstein-Barr virus. 40 years on. Nat Rev Cancer. 2004:4:757-68.

2. Zuo L, Yue W, Du S, Xin S, Zhang J, Liu L, et al. An update: Epstein-Barr virus and immune evasion via microRNA regulation. Virol Sin. 2017:32:175-87.

3. Lu JH, Tang YL, Yu HB, Zhou JH, Fu CY, Zeng X, et al. Epstein-Barr virus facilitates the malignant potential of immortalized epithelial cells: from latent genome to viral production and maintenance. Lab Investig. 2010;90:196-209.

4. Carninci P, Kasukawa T, Katayama S, Gough J, Frith MC, Maeda N, et al. The transcriptional landscape of the mammalian genome. Science. 2005;309:1559-63.

5. Xie Y, Dang W, Zhang S, Yue W, Yang L, Zhai X, et al. The role of exosomal noncoding RNAs in cancer. Mol Cancer. 2019;18:37.

6. Guttman M, Amit I, Garber M, French C, Lin MF, Feldser D, et al. Chromatin signature reveals over a thousand highly conserved large non-coding RNAs in mammals. Nature. 2009;458:223-7.

7. Shi L, Peng F, Tao Y, Fan X, Li N. Roles of long noncoding RNAs in hepatocellular carcinoma. Virus Res. 2016;223:131-9.

8. Damas ND, Fossat N. Functional interplay between RNA viruses and noncoding RNA in mammals. Noncoding RNA. 2019;5:7.

9. Ahmed W, Liu ZF. Long non-coding RNAs: novel players in regulation of immune response upon herpesvirus infection. Front Immunol. 2018;9:761.

10. Fan Q, Wei S, Yang L, Zhang X, Peng X, Su D, et al. The emerging role of exosomederived non-coding RNAs in cancer biology. Cancer Lett. 2018;414:107-15.

11. Verhoeven RJ, Tong S, Zhang G, Zong J, Chen Y, Jin DY. NF-kappaB signaling regulates expression of Epstein-Barr virus BART MicroRNAs and long noncoding RNAs in nasopharyngeal carcinoma. J Virol. 2016;90:6475-88.

12. Ponting $\mathrm{CP}$, Oliver $\mathrm{PL}$, Reik W. Evolution and functions of long noncoding RNAs. Cell. 2009;136:629-41.

13. Li XX, Liang XJ, Zhou LY, Liu RJ, Bi W, Zhang S, Li SS, Yang WH, Chen ZC, Yang XM, Zhang PF. Analysis of differential expressions of long non-coding RNAs in nasopharyngeal carcinoma using next-generation deep sequencing. J Cancer. 2018;9:1943-50.

14. He B, Li W, Wu Y, Wei F, Gong Z, Bo H, Wang Y, Li X, Xiang B, Guo C, et al. EpsteinBarr virus-encoded miR-BART6-3p inhibits cancer cell metastasis and invasion by targeting long non-coding RNA LOC553103. Cell Death Dis. 2016;7:e2353.

15. He B, Zeng J, Chao W, Chen X, Huang Y, Deng K, Huang Z, Li J, Dai M, Chen $S$, et al. Serum long non-coding RNAs MALAT1, AFAP1-AS1 and AL359062 as diagnostic and prognostic biomarkers for nasopharyngeal carcinoma. Oncotarget. 2017;8:41166-77.

16. Liu J, Yang C, Gu Y, Li C, Zhang H, Zhang W, Wang X, Wu N, Zheng C. Knockdown of the IncRNA SNHG8 inhibits cell growth in Epstein-Barr virusassociated gastric carcinoma. Cell Mol Biol Lett. 2018;23:17.

17. Jing JJ, Wang ZY, Li H, Sun LP, Yuan Y. Key elements involved in EpsteinBarr virus-associated gastric cancer and their network regulation. Cancer Cell Int. 2018;18:146.

18. Zhang J, Zhang S, Zuo L, Yue W. Differential expression profiling of IncRNAs related to Epstein-Barr virus infection in the epithelial cells. J Med Virol. 2019:91:1845-55.

19. Wang QM, Lian GY, Song Y, Huang YF, Gong Y. LncRNA MALAT1 promotes tumorigenesis and immune escape of diffuse large B cell lymphoma by sponging miR-195. Life Sci. 2019;231:116335.

20. Wang C, Li D, Zhang L, Jiang S, Liang J, Narita Y. RNA sequencing analyses of gene expression during Epstein-Barr Virus infection of primary B lymphocytes. J Virol. 2019;93. 
21. Gallo A, Vella S, Miele M, Timoneri F, Di Bella M, Bosi S, Sciveres M, Conald PG. Global profiling of viral and cellular non-coding RNAs in Epstein-Barr virus-induced lymphoblastoid cell lines and released exosome cargos. Cancer Lett. 2017:388:334-43.

22. Sethuraman S, Thomas M, Gay LA, Renne R. Computational analysis of ribonomics datasets identifies long non-coding RNA targets of gammaherpesviral miRNAs. Nucleic Acids Res. 2018;46:8574-89.

23. Yu H, Lu J, Zuo L, Yan Q, Yu Z, Li X, Huang J, Zhao L, Tang H, Luo Z, et al. Epstein-Barr virus downregulates microRNA 203 through the oncoprotein latent membrane protein 1: a contribution to increased tumor incidence in epithelial cells. J Virol. 2012;86:3088-99.

24. Zuo LL, Zhang J, Liu LZ, Zhou Q, Du SJ, Xin SY, Ning ZP, Yang J, Yu HB, Yue WX, et al. Cadherin 6 is activated by Epstein-Barr virus LMP1 to mediate EMT and metastasis as an interplay node of multiple pathways in nasopharyngeal carcinoma. Oncogenesis. 2017:6:402.

25. Chang ET, Adami HO. The enigmatic epidemiology of nasopharyngeal carcinoma. Cancer Epidemiol Biomarkers Prev. 2006;15:1765-77.

26. Liu L, Zuo L, Yang J, Xin S, Zhang J, Zhou J, Li G, Tang J, Lu J. Exosomal cyclophilin $\mathrm{A}$ as a novel noninvasive biomarker for Epstein-Barr virus associated nasopharyngeal carcinoma. Cancer Med. 2019;8:3142-51.

27. Zheng X, Wang J, Wei L, Peng Q, Gao Y, Fu Y, Lu Y, Qin Z, Zhang X, Lu J, et al. Epstein-Barr virus microRNA miR-BART5-3p inhibits p53 expression. J Virol. 2018:92.

28. Zhang W, Huang C, Gong Z, Zhao Y, Tang K, Li X, Fan S, Shi L, Li X, Zhang $P$, et al. Expression of LINC00312, a long intergenic non-coding RNA, is negatively correlated with tumor size but positively correlated with lymph node metastasis in nasopharyngeal carcinoma. J Mol Histol. 2013:44:545-54.

29. Lu Y, Qin Z, Wang J, Zheng X, Lu J, Zhang X, Wei L, Peng Q, Zheng Y, Ou C, et al. Epstein-Barr virus miR-BART6-3p inhibits the RIG-I pathway. J Innate Immun. 2017;9:574-86.

30. Liu L, Zhou Q, Xie Y, Zuo L, Zhu F, Lu J. Extracellular vesicles: novel vehicles in herpesvirus infection. Virol Sin. 2017:32:349-56.

31. Zuo L, Xie Y, Tang J, Xin S, Liu L, Zhang S, Yan Q, Zhu F, Lu J. Targeting exosomal EBV-LMP1 transfer and miR-203 expression via the NF-kappaB pathway: the therapeutic role of aspirin in NPC. Mol Ther Nucleic Acids. 2019;17:175-84.

32. Yue W, Zhu M, Zuo L, Xin S, Zhang J, Liu L, Li S, Dang W, Zhang S, Xie Y, et al. Early pattern of Epstein-Barr virus infection in gastric epithelial cells by "cell-in-cell". Virol Sin. 2019;34:253-61.

33. Cao WJ, Wu HL, He BS, Zhang YS, Zhang ZY. Analysis of long non-coding RNA expression profiles in gastric cancer. World J Gastroenterol. 2013;19:3658-64.

34. Huang T, Ji Y, Hu D, Chen B, Zhang H, Li C, Chen G, Luo X, Zheng XW, Lin $X$. SNHG8 is identified as a key regulator of Epstein-Barr virus (EBV)associated gastric cancer by an integrative analysis of IncRNA and mRNA expression. Oncotarget. 2016;7:80990-1002.

35. Munschauer M, Nguyen $C T$, Sirokman $K$, Hartigan CR, Hogstrom L, Engreitz JM, Ulirsch JC, Fulco CP, Subramanian V, Chen J, et al. The NORAD IncRNA assembles a topoisomerase complex critical for genome stability. Nature. 2018;561:132-6,

36. Wu J, Zhang H, Zheng Y, Jin X, Liu M, Li S, Zhao Q, Liu X, Wang Y, Shi M, et al. The long noncoding RNA MALAT1 induces tolerogenic dendritic cells and regulatory T cells via miR155/dendritic cell-specific intercellular sdhesion molecule-3 grabbing nonintegrin/L10 axis. Front Immunol. 2018:9:1847.

37. Park R, Miller G. Epstein-Barr virus-induced nodules on viral replication compartments contain RNA processing proteins and a viral long noncoding RNA. J Virol. 2018;92:20.

38. Qiu J, Smith P, Leahy L, Thorley-Lawson DA. The Epstein-Barr virus encoded BART miRNAs potentiate tumor growth in vivo. PLoS Pathog. 2015;11:e1004561.

39. Edwards RH, Marquitz AR, Raab-Traub N. Epstein-Barr virus BART microRNAs are produced from a large intron prior to splicing. J Virol. 2008:82:9094-106.

40. Marquitz AR, Mathur A, Edwards RH, Raab-Traub N. Host gene expression is regulated by two types of noncoding RNAs transcribed from the EpsteinBarr virus BamHI a rightward transcript region. J Virol. 2015;89:11256-68.

41. Kang GH, Lee S, Kim WH, Lee HW, Kim JC, Rhyu MG, et al. Epstein-barr viruspositive gastric carcinoma demonstrates frequent aberrant methylation of multiple genes and constitutes CpG island methylator phenotype-positive gastric carcinoma. Am J Pathol. 2002;160:787-94.

42. Rennekamp AJ, Lieberman PM. Initiation of Epstein-Barr virus Iytic replication requires transcription and the formation of a stable RNA-DNA hybrid molecule at Orilyt. J Virol. 2011;85:2837-50.

\section{Publisher's Note}

Springer Nature remains neutral with regard to jurisdictional claims in published maps and institutional affiliations.

\section{Ready to submit your research? Choose BMC and benefit from:}

- fast, convenient online submission

- thorough peer review by experienced researchers in your field

- rapid publication on acceptance

- support for research data, including large and complex data types

- gold Open Access which fosters wider collaboration and increased citations

- maximum visibility for your research: over $100 \mathrm{M}$ website views per year

At BMC, research is always in progress.

Learn more biomedcentral.com/submissions 